\title{
Association between Albuminuria, Carotid Atherosclerosis, Arterial Stiffness, and Peripheral Arterial Disease in Korean Type 2 Diabetic Patients
}

\author{
Seong-Woo Choi ${ }^{a}$ Woo-Jun Yun ${ }^{a}$ Hey-Yeon Kim ${ }^{b}$ Young-Hoon Lee ${ }^{c}$ \\ Sun-Seog Kweon ${ }^{\text {a,d }}$ Jung-Ae Rhee ${ }^{a}$ Jin-Su Choi ${ }^{a}$ Min-Ho Shin ${ }^{a}$ \\ a Department of Preventive Medicine, Chonnam National University Medical School, Gwangju, \\ ${ }^{b}$ Department of Preventive Medicine, Seonam University College of Medicine, Namwon, \\ 'Gwangju-Jeonnam Regional Cardiocerebrovascular Center, Chonnam National University Hospital, \\ Gwangju, and d Jeonnam Regional Cancer Center, Chonnam National University Hwasun Hospital, \\ Hwasun-gun, Republic of Korea
}

\section{Key Words}

Albuminuria · Arterial stiffness - Carotid atherosclerosis .

Korean type 2 diabetic patients $\cdot$ Peripheral arterial disease

\begin{abstract}
Background: To evaluate the association between albuminuria, carotid atherosclerosis, arterial stiffness, and peripheral arterial disease (PAD) in Korean type 2 diabetic patients. Methods: In total, 673 type 2 diabetic patients registered with the public health center participated. Following an overnight fast, venous blood and urine samples were collected and analyzed. The carotid intima-media thickness (IMT), amount of carotid plaque, brachial ankle pulse wave velocity (baPWV), and the ankle-brachial index of each patient were also assessed. Results: Albuminuria was significantly associated with PAD (odds ratio (OR) 2.33; 95\% confidence interval $(\mathrm{Cl}) 1.28-4.25$ for normoalbuminuria vs. microalbuminuria and $\mathrm{OR} 3.28 ; 95 \% \mathrm{Cl} 1.40-7.66$ for normoalbuminuria vs. macroalbuminuria), but not with carotid plaque. The mean baPWV differed significantly according to the level of albumin relative to the creatinine ratio $(1,764.79$,
\end{abstract}

$1,778.98$, and 2,001.33, respectively; $p<0.001$ ), while no significant difference was observed in the mean IMT value $(0.73$, 0.74 , and 0.72 , respectively; $p=0.399$ ). Conclusions: Albuminuria was significantly associated with baPWV and PAD, but not with carotid plaque or CCA-IMT, in Korean type 2 diabetic patients.

Copyright $\odot 2010$ S. Karger AG, Basel

\section{Introduction}

In the 20th century, cardiovascular disease (CVD) became the main cause of mortality and morbidity in Western populations, and in 2000, the global prevalence of diabetes was estimated at 171 million [1]. The risk of coronary artery disease is six times higher in type 2 diabetes patients than in the general population $[2,3]$. Therefore, the development of methods to estimate the risk of CVD in diabetes patients is highly desirable and will aid in preventing CVD incidence and lowering the disease burden of diabetes.

\section{KARGER}

Fax +4161306 1234 E-Mail karger@karger.ch www.karger.com
(C) 2010 S. Karger AG, Basel

$1420-4096 / 10 / 0332-0111 \$ 26.00 / 0$

Accessible online at:

www.karger.com/kbr
Min-Ho Shin

Department of Preventive Medicine

Chonnam National University Medical School, 5, Hak-1-dong

Dong-gu, Gwangju 501-746 (Republic of Korea)

Tel. +82 62220 4166, Fax +82 62233 0305, E-Mail mhshinx@ paran.com 
One of these measures, albuminuria, is correlated with cardiovascular mortality and morbidity, especially in high-risk groups, diabetic patients [4] and hypertensive patients [5]. It has also been reported that microalbuminuria and macroalbuminuria are predictors of mortality [6] and end-organ damage [7]. Because albuminuria is easily measurable, it can be broadly applied in the routine care of diabetic patients.

Many surrogate measures for CVD that estimate subclinical atherosclerosis have been developed. Carotid artery intima-media thickness (IMT) is known to be correlated with coronary artery disease, stroke, and several other risk factors [8-10]. Carotid plaque has different pathogenic characteristics to IMT; however, both IMT and carotid plaque share a common association with atherosclerosis and ischemic heart symptoms [11, 12]. The ankle-brachial index (ABI) is an easy method for measuring peripheral arterial disease (PAD), therefore $\mathrm{ABI}$ is commonly used to screen for PAD in diabetics [13]. Pulse wave velocity (PWV) has also been identified as a strong independent predictor of cardiovascular risk [14].

Many studies have evaluated the association between albuminuria and these surrogate measures [15-18]. Most researchers, however, have examined the association between albuminuria and only one or two variables. Therefore, it is difficult to generally assess the association between albuminuria and various subclinical atherosclerosis phenotypes.

In contrast to previous studies that only measured one or two variables, we measured the albumin to creatinine ratio (ACR), common carotid artery (CCA)-IMT, carotid plaque, brachial ankle PWV (baPWV), and ABI in our subjects and examined whether albuminuria would be correlated with carotid atherosclerosis, arterial stiffness, and PAD in Korean type 2 diabetic patients.

\section{Subjects and Methods}

\section{Subjects}

Among the 1,275 type 2 diabetes patients registered at the Public Health Center of Seo-gu, Gwangju, and Gokseng-gun, Jeollanamdo, Korea, 709 subjects with type 2 diabetes participated in this study. The response rate for this study was $55.5 \%$. Ten patients who did not provide a blood sample and 26 patients who did not provide a urine sample were excluded from the study. After excluding these patients, a total of 673 patients participated in the study. This study was conducted in accordance with the Declaration of Helsinki guidelines. The study protocol was approved by the Institutional Review Board of Chonnam National University Hospital, and informed consent was obtained from each subject.

\section{Methods}

Well-trained examiners interviewed the patients using a survey that included questions on cigarette use, alcohol consumption, physical activity, antidiabetic medication use, antihypertension medication use, and CVD history.

Weight was measured to the nearest $0.1 \mathrm{~kg}$ while the subjects were dressed in light clothes and height was measured to the nearest $0.1 \mathrm{~cm}$ in stocking feet. Abdominal circumference was measured to the nearest $0.1 \mathrm{~cm}$ at expiration through a horizontal plane around the abdomen midway between the lowest rib and iliac crest. Blood pressure (BP) was measured twice using a standard mercury sphygmomanometer after the subjects had rested for at least $5 \mathrm{~min}$. BP measurements were read to the nearest $2 \mathrm{~mm} \mathrm{Hg}$.

Following an overnight fast, venous blood was collected and the serum was separated on site and stored at $-70^{\circ} \mathrm{C}$ until further analysis. Total cholesterol, high-density lipoprotein (HDL) cholesterol, and triglyceride concentrations were analyzed using enzymatic methods. All sera were examined using an automatic analyzer (Hitachi-7600, Hitachi Ltd, Tokyo, Japan). Low-density lipoprotein (LDL) cholesterol was measured as proposed by Friedewald et al. [19], except when triglyceride levels exceeded 400 $\mathrm{mg} / \mathrm{dl}$. In such instances, the data were treated as missing.

Urinary albumin and creatinine concentrations were measured using a turbidimetric immunoassay and the Jaffe method [20] on an automated analyzer (Hitachi-7600, Hitachi Ltd). ACR was calculated by dividing the urinary albumin concentration in micrograms by the urinary creatinine concentration in milligrams. An ACR $<30.0 \mu \mathrm{g} / \mathrm{mg}$ creatinine was defined as normoalbuminuria, an ACR from 30 to $299.9 \mu \mathrm{g} / \mathrm{mg}$ creatinine was defined as microalbuminuria, and an ACR $\geq 300.0 \mu \mathrm{g} / \mathrm{mg}$ creatinine was defined as macroalbuminuria. The kidney function was ascertained by estimated glomerular filtration rate, which was calculated by the Modification of Diet in Renal Disease (MDRD) formula [21]:

$186.3 \times\left(\right.$ serum creatinine $\left.\mathrm{e}^{-1.154}\right) \times\left(\right.$ age $\left.^{-0.203}\right) \times$

0.742 (if female),

where serum creatinine concentration is in $\mathrm{mg} / \mathrm{dl}$.

Well-trained medical doctors performed ultrasonographic scans of the carotid arteries using high-resolution B-mode ultrasound (SonoAce 9900, Medison, Korea) with an electrical linear array transducer $(7.5 \mathrm{MHz})$. IMT was defined as the distance from the leading edge of the first echogenic line to the second echogenic line, which indicated the media-adventitia interface. Images of the thickest point within $10 \mathrm{~mm}$ from the carotid bulb to the CCA were saved as CCA-IMT, and then measured using SigmaScan Pro Version 5.0.0 (SPSS Inc., Chicago, Ill., USA).

The examiners evaluated the CCA, carotid bulb, and internal carotid artery to determine the amount of carotid plaque. Protrusions into the lumen that were $100 \%$ thicker than the nearest area were defined as plaque. If the plaque was the thickest point, the distance to the nearest point without plaque was defined as the IMT.

After at least a 5-min rest, the ABI and baPWV were calculated automatically in the supine position using the VP-1000 system (Colin Co., Komaki, Japan) with cuffs around both arms and ankles. If any of the ABIs were $<0.9$, the patient was defined as having PAD. 
Table 1. General and biochemical characteristics

\begin{tabular}{|c|c|c|c|c|c|}
\hline \multirow[t]{2}{*}{ Variable } & \multicolumn{3}{|c|}{ ACR, $\mu \mathrm{g} / \mathrm{mg}$ creatinine } & \multirow[t]{2}{*}{ Total } & \multirow[t]{2}{*}{$\mathrm{p}$ value } \\
\hline & $<30$ & $30-299$ & $\geq 300$ & & \\
\hline Number (\%) & $355(52.7)$ & $260(38.6)$ & $58(8.6)$ & $673(100)$ & - \\
\hline ACR, $\mu \mathrm{g} / \mathrm{mg}$ creatinine & $16.0 \pm 6.6$ & $81.2 \pm 59.6$ & $876.1 \pm 648.5$ & $115.3 \pm 304.4$ & $<0.001$ \\
\hline $\mathrm{eGFR}, \mathrm{ml} / \mathrm{min} / 1.73 \mathrm{~m}^{2}$ & $66.1 \pm 13.6$ & $65.5 \pm 17.2$ & $55.6 \pm 21.6$ & $65.0 \pm 16.1$ & $<0.001$ \\
\hline Male (\%) & $103(29.0)$ & $93(35.8)$ & $19(32.8)$ & $215(31.9)$ & 0.165 \\
\hline Age, years & $67.7 \pm 9.7$ & $68.6 \pm 10.2$ & $68.9 \pm 11.1$ & $68.2 \pm 10.0$ & 0.470 \\
\hline Age at diabetic diagnosis, years & $59.7 \pm 11.7$ & $59.5 \pm 12.8$ & $56.2 \pm 14.2$ & $59.3 \pm 12.4$ & 0.131 \\
\hline Diabetic duration, years & $8.0 \pm 7.6$ & $9.1 \pm 7.9$ & $12.7 \pm 10.2$ & $8.9 \pm 8.0$ & $<0.001$ \\
\hline Height, $\mathrm{cm}$ & $154.8 \pm 8.3$ & $154.8 \pm 8.9$ & $154.0 \pm 8.1$ & $154.7 \pm 8.5$ & 0.799 \\
\hline Weight, kg & $58.6 \pm 9.8$ & $58.6 \pm 9.7$ & $57.4 \pm 11.1$ & $58.5 \pm 9.9$ & 0.665 \\
\hline $\mathrm{BMI}$ & $24.5 \pm 3.9$ & $24.5 \pm 3.5$ & $24.2 \pm 4.1$ & $24.5 \pm 3.7$ & 0.795 \\
\hline Abdomen circumference, $\mathrm{cm}$ & $88.5 \pm 9.3$ & $89.3 \pm 9.0$ & $88.6 \pm 11.0$ & $88.8 \pm 9.4$ & 0.631 \\
\hline Systolic BP, mm Hg & $126.8 \pm 16.4$ & $132.7 \pm 17.4$ & $141.8 \pm 17.8$ & $130.4 \pm 17.5$ & $<0.001$ \\
\hline Diastolic BP, mm Hg & $71.4 \pm 9.4$ & $72.6 \pm 10.2$ & $73.8 \pm 10.6$ & $72.1 \pm 9.9$ & 0.114 \\
\hline Total cholesterol, mg/dl & $191.2 \pm 42.2$ & $196.3 \pm 44.2$ & $197.8 \pm 52.9$ & $193.7 \pm 44.0$ & 0.284 \\
\hline Triglycerides, $\mathrm{mg} / \mathrm{dl}$ & $176.6 \pm 77.8$ & $188.0 \pm 107.0$ & $195.5 \pm 86.6$ & $182.6 \pm 91.0$ & 0.168 \\
\hline HDL cholesterol, mg/dl & $47.9 \pm 11.7$ & $47.3 \pm 11.5$ & $47.6 \pm 12.6$ & $47.6 \pm 11.7$ & 0.831 \\
\hline LDL cholesterol, mg/dl & $108.1 \pm 34.1$ & $112.9 \pm 34.5$ & $111.0 \pm 42.7$ & $110.2 \pm 35.1$ & 0.246 \\
\hline Fasting plasma glucose, $\mathrm{mg} / \mathrm{dl}$ & $129.2 \pm 45.8$ & $144.7 \pm 53.6$ & $150.3 \pm 55.7$ & $137.0 \pm 50.5$ & $<0.001$ \\
\hline $\mathrm{HbA}_{1 \mathrm{c}}, \%$ & $7.2 \pm 1.6$ & $7.8 \pm 1.5$ & $7.8 \pm 1.9$ & $7.5 \pm 1.6$ & $<0.001$ \\
\hline CCA-IMT, mm & $0.73 \pm 0.15$ & $0.75 \pm 0.16$ & $0.73 \pm 0.15$ & $0.74 \pm 0.15$ & 0.162 \\
\hline $\mathrm{baPWV}, \mathrm{m} / \mathrm{s}^{\mathrm{a}}$ & $1,722.9 \pm 381.1$ & $1,841.3 \pm 388.3$ & $2,037.9 \pm 525.6$ & $1,796.3 \pm 408.2$ & $<0.001$ \\
\hline Current smoker (\%) & $52(14.6)$ & $43(16.7)$ & $11(19.0)$ & $106(15.8)$ & 0.334 \\
\hline Hypertension $(\%)^{\mathrm{b}}$ & $241(68.3)$ & $194(75.2)$ & $52(89.7)$ & $487(72.8)$ & 0.001 \\
\hline Numbers of carotid plaques $\geq 1(\%)$ & $157(44.9)$ & $131(51.4)$ & $33(57.9)$ & $321(48.5)$ & 0.030 \\
\hline Oral diabetics $(\%)$ & $323(96.4)$ & $237(91.9)$ & $49(86.0)$ & $609(93.7)$ & 0.047 \\
\hline Insulin injection (\%) & $12(3.6)$ & $21(8.1)$ & $8(14.0)$ & $41(6.3)$ & 0.047 \\
\hline $\mathrm{ABI} \leq 0.9(\%)$ & $21(6.0)$ & $39(15.1)$ & $13(22.4)$ & $73(10.9)$ & $<0.001$ \\
\hline
\end{tabular}

Values are given as the mean $\pm \mathrm{SD}$ or number (\%). ACR $=$ Albumin to creatinine ratio; eGFR = estimated glomerular filtration rate; $\mathrm{BMI}=$ body mass index; $\mathrm{BP}=$ blood pressure; $\mathrm{HDL}=$ high-density lipoprotein; $\mathrm{LDL}=$ low-density lipoprotein; $\mathrm{HbA}_{1 \mathrm{c}}=$ glycated hemoglobin; CCA = common carotid artery; IMT = intima-media thickness; baPWV = brachial-ankle pulse wave velocity; ABI = ankle-brachial index.

${ }^{a}$ Calculated with the patients with their $\mathrm{ABI} \geq 0.9$ after excluding the patients with $\mathrm{ABI}<0.9$.

${ }^{\mathrm{b}}$ Hypertension was defined as systolic blood pressure $\geq 130 \mathrm{~mm}$ Hg or diastolic blood pressure $\geq 80 \mathrm{~mm}$ Hg or taking antihypertension medication.

\section{Statistics}

Data are given as mean \pm SD or percentage for categorical variables. Statistical significance was set at $\mathrm{p}<0.05$. Statistical analysis was performed using the SPSS 15.0 software package (SPSS Inc.). Plaque was dichotomized according to the number of plaques ( $\leq 1$ or $>1$ ), while PAD was dichotomized according to the ABI value ( $\leq 0.9$ or $>0.9$ ). A logistic regression was used to provide odds ratios (ORs) for the categorical variables, i.e. carotid plaque and PAD, according to the ACR level (ACR $<30,30-300$, and ACR $\geq 300 \mu \mathrm{g} / \mathrm{mg}$ creatinine). An analysis of covariance was used to compare the means of the continuous variables, i.e. CCA-IMT and PWV, according to the ACR level. When we calculated PWV, we excluded any subjects that had ABIs $<0.9$ or who had a history of CVD. We defined hypertension as systolic BP $\geq 140 \mathrm{~mm} \mathrm{Hg}$, diastolic BP $\geq 90 \mathrm{~mm} \mathrm{Hg}$, or taking antihypertension medication.

\section{Results}

\section{General and Biochemical Characteristics}

General and biochemical characteristics of the 673 subjects (215 men and 458 women) are detailed in table 1. $355(52.7 \%)$ subjects had an ACR $<30 \mu \mathrm{g} / \mathrm{mg}$ creatinine, 260 (38.6\%) had an ACR between 30 and $300 \mu \mathrm{g} / \mathrm{mg}$ creatinine, and 58 (8.6\%) had an ACR $\geq 300 \mu \mathrm{g} / \mathrm{mg}$ creatinine. Diabetic duration, systolic BP, fasting plasma glucose, $\mathrm{HbA}_{1 \mathrm{c}}$, and baPWV tended to increase in groups with higher ACR levels. In contrast, estimated glomerular filtration rate tended to decrease in groups with higher ACR levels. Patients with higher ACR levels were more 
Table 2. ORs for carotid plaque and PAD according to ACR level

\begin{tabular}{lllll}
\hline & ACR, $\mu \mathrm{g} / \mathrm{mg}$ creatinine & Crude OR & Model $1^{\mathrm{a}}$ & ${\text { Model } 2^{\mathrm{b}}}^{\mathrm{a}}$ \\
\hline Carotid plaque $^{\mathrm{c}}$ & $<30$ & 1.00 & 1.00 & 1.00 \\
& $30-299$ & $1.30(0.94,1.79)$ & $1.21(0.87,1.69)$ & $1.06(0.74,1.53)$ \\
& $\geq 300$ & $1.69(0.96,2.98)$ & $1.61(0.90,2.89)$ & $1.36(0.72,2.55)$ \\
& p for the trend & 0.030 & 0.077 & 0.396 \\
\hline $\mathrm{PAD}^{\mathrm{d}}$ & $<30$ & 1.00 & 1.00 & 1.00 \\
& $30-299$ & $2.78(1.59,4.85)$ & $2.57(1.46,4.52)$ & $2.33(1.28,4.25)$ \\
& $\geq 300$ & $4.53(2.12,9.66)$ & $4.27(1.96,9.26)$ & $3.28(1.40,7.66)$ \\
& p for the trend & $<0.001$ & $<0.001$ & 0.001 \\
\hline
\end{tabular}

$\mathrm{PAD}=$ Peripheral arterial disease; $\mathrm{ACR}=$ albumin to creatinine ratio.

${ }^{\text {a }}$ Adjusted by sex, age.

b Adjusted by sex, age, BMI, smoking, diabetic duration, systolic BP, HDL, LDL, triglyceride, fasting blood glucose, and $\mathrm{HbA}_{1 \mathrm{c}}$.

${ }^{\mathrm{c}}$ Carotid plaque was dichotomized according to the number of plaques $(\leq 1$ or $>1)$.

${ }^{\mathrm{d}} \mathrm{PAD}$ was dichotomized according to $\mathrm{ABI} \leq 0.9$ or $\mathrm{ABI}>0.9$.

likely to be hypertensive, have more plaque in their carotid arteries, and have a higher percentage of their ABIs $\leq 0.9$.

\section{ORs for Carotid Plaque and PAD according to ACR}

Level

The ORs for carotid plaque and PAD according to ACR level are listed in table 2 . When adjusted by sex and age, albuminuria was not significantly associated with carotid plaque, but was significantly associated with PAD (OR 2.57; 95\% confidence interval (CI) 1.46-4.52 for normoalbuminuria vs. microalbuminuria and OR 4.27; $95 \%$ CI 1.96-9.26 for normoalbuminuria vs. macroalbuminuria). When additionally adjusted for other covariates (i.e. BMI, smoking, diabetic duration, systolic BP, HDL, $\mathrm{LDL}$, triglyceride, fasting blood glucose, and $\mathrm{HbA}_{1 \mathrm{c}}$ ), albuminuria was not significantly associated with plaque; however, the significant association between albuminuria and PAD was slightly attenuated (OR 2.33; 95\% CI 1.28-4.25 for normoalbuminuria vs. microalbuminuria and OR 3.28; 95\% CI 1.40-7.66 for normoalbuminuria vs. macroalbuminuria).

\section{Comparison between IMT and baPWV Means according to ACR Level}

The means and standard errors for CCA-IMT and baPWV according to ACR level are listed in table 3 . The mean CCA-IMT value was not significantly different according to ACR level $(0.73,0.74,0.72$, and $p=0.399)$; however, a significant difference was found in the mean
baPWV value according to ACR level after adjusting for other covariates $(1,764.79,1,778.98,2,001.33$, and $\mathrm{p}<$ $0.001)$.

\section{Discussion}

We evaluated the association between albuminuria, carotid atherosclerosis, arterial stiffness, and PAD in Korean type 2 diabetics. We found that ACR level was strongly associated with PAD and baPWV, when adjusted for other CVD risk factors, such as gender, age, BMI, smoking, diabetic duration, systolic BP, HDL, LDL, triglyceride, fasting blood glucose, and $\mathrm{HbA}_{1 c}$. No significant association, however, was observed between ACR levels, carotid plaque, and CCA-IMT.

In this study, after adjusting for other covariates, ACR levels were not significantly associated with carotid plaque (OR 1.36; 95\% CI 0.72-2.55 for normoalbuminuria and macroalbuminuria) or the mean CCA-IMT value $(0.73,0.74$, and $0.72 ; \mathrm{p}=0.399)$. In a cross-sectional study of 368 participants [17], researchers divided the participants into two groups according to urine albumin excretion (UAE): normoalbuminuria (UAE $<20 \mu \mathrm{g} / \mathrm{min}$ ) and microalbuminuria (UAE $20-200 \mu \mathrm{g} / \mathrm{mg}$ ). When they compared the number of carotid plaques, no significant difference was found between subjects with normoalbuminuria and microalbuminuria. In another study of 830 subjects, Ishizaka et al. [22] reported that albuminuria was positively associated with carotid IMT (OR 2.21; 
Table 3. Comparison between IMT and baPWV means according to ACR level

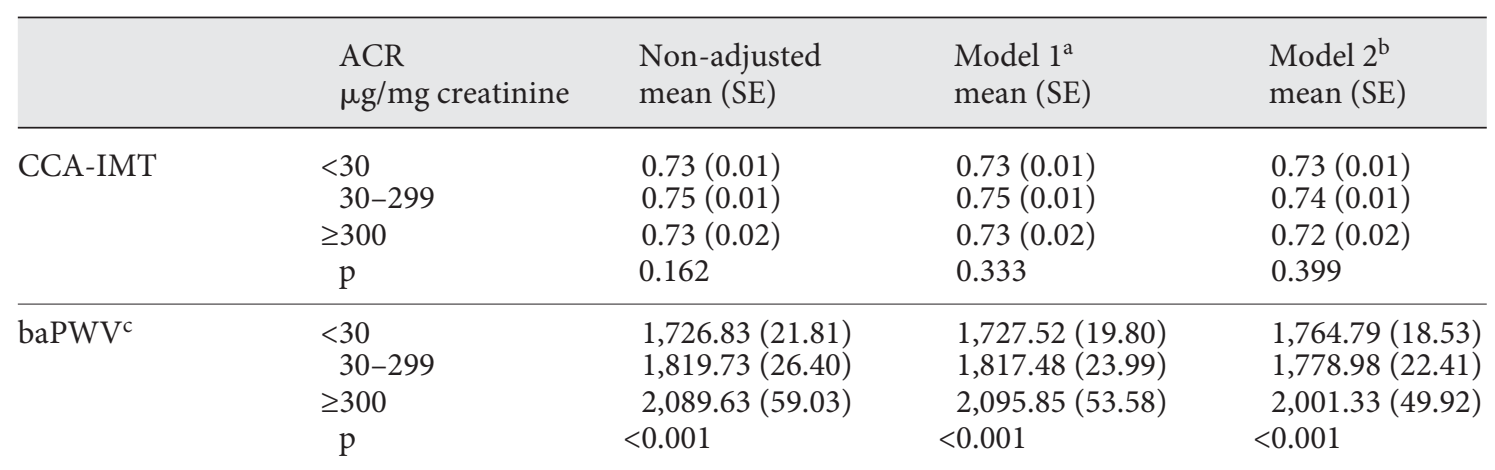

$\mathrm{CCA}=$ Common carotid artery; IMT = intima-media thickness; baPWV = brachial-ankle pulse wave velocity.

a Adjusted by sex, age.

b Adjusted by sex, age, BMI, smoking, diabetic duration, systolic BP, HDL, LDL, triglyceride, fasting blood glucose, and $\mathrm{HbA}_{1 \mathrm{c}}$.

${ }^{c}$ Calculated with the patients with their $\mathrm{ABI} \geq 0.9$ after excluding the patients with $\mathrm{ABI}<0.9$.

95\% CI 1.15-4.23); however, this association disappeared after adjusting for age (OR 1.26; 95\% CI 0.61-2.61). Similar results have been published for diabetic patients. Consistent with our results, in a study of 588 type 2 diabetic patients, investigators estimated the association between $\log (\mathrm{ACR})$ and carotid IMT, but $\log (\mathrm{ACR})$ was not associated with carotid IMT $(\mathrm{p}=0.1194)$ [23].

Based on our results, albuminuria was not associated with carotid plaque or IMT. There may be several explanations for this. First, the diabetic state itself has such a powerful influence on carotid plaque and CCA-IMT that any effect of albuminuria is masked. In diabetics, it has been suggested that the endothelium is stressed by advanced glycosylation end products, leading to increased albumin permeability [24], and that disturbed proteoglycan synthesis causes the glomerular membrane to lose its charge sensitivity and integrity [25]. Second, because carotid plaque formation and progression commonly occur at places of non-laminar turbulent flow, such as the proximal internal carotid artery or at the bifurcation [26], measures of CCA-IMT might not be representative of what is actually occurring at more atherosclerosis-prone locations [27].

We found that mean baPWV values were significantly different according to ACR level after adjusting for potentially confounding variables $(1,764.79,1,778.98$, and $2,001.33$, respectively; $p<0.001$ ). In a study of 346 type 2 diabetic patients [15], researchers investigated the association between baPWV and ACR. They categorized patients into three groups, according to ACR level: normo- albuminuria ( $<30 \mu \mathrm{g} / \mathrm{mg}$ creatinine), microalbuminuria (30-299 $\mu \mathrm{g} / \mathrm{mg}$ creatinine), and macroalbuminuria ( $\geq 300 \mu \mathrm{g} / \mathrm{mg}$ creatinine). They found that baPWV was significantly higher in patients with macroalbuminuria or microalbuminuria, compared with normoalbuminuria patients. Additionally, ACR was significantly associated with baPWV $(\mathrm{r}=0.24 ; \mathrm{p}<0.0001)$ and was an independent risk factor for baPWV. In a study of 167 type 2 diabetic patients, Ishimura et al. [28] reported that aortic PWV was significantly correlated with $\log (\mathrm{UAE})$ ( $\mathrm{r}=$ $0.269 ; \mathrm{p}<0.0001)$. In a multiple regression analysis, $\log$ (UAE) was an independent risk factor for aortic PWV after adjustment for other CV factors $\left(\mathrm{R}^{2}=0.246\right.$; $\mathrm{p}<$ 0.0001).

Arterial stiffness is a major contributor to CVD [29] and aortic PWV is one of a few indices of arterial stiffness that has been directly linked with cardiovascular mortality and morbidity [30]. However, baPWV is still a relatively new measure of arterial stiffness [31], compared with aortic PWV, and they are differentiated in that baPWV reflects a combination of aortic and peripheral stiffness [32]. Nevertheless, at both clinical and subclinical levels, baPWV correlates with the degree of atherosclerotic vascular damage [33], similar to aortic PWV.

A possible explanation for the mechanism linking arterial stiffness and albuminuria is that increased arterial stiffness is a major cause of a wide pulse pressure and systolic hypertension [34]. An increase in arterial pulse pressure could contribute to glomerular damage, because kidney cells have no protection against pulsatile stress [35]. 
Hypertension has been significantly associated with microalbuminuria [36], because systemic BP affects the loss of albumin from the renal glomerulus. However, in a study of 136 community residents [37], after adjusting for other covariates, including systolic BP and pulse pressure, microalbuminuria was independently associated with $\mathrm{PWV}$, indicating that the association was not merely a reflection of the increases in pulse pressure and systolic BP.

Similar to our results, several studies have shown an association between ACR and PAD (OR 2.33; 95\% CI $1.28-4.25$ for normoalbuminuria vs. microalbuminuria and OR 3.28; 95\% CI 1.40-7.66 for normoalbuminuria vs. macroalbuminuria). For example, in a cross-sectional study of 3,312 subjects, researchers investigated the ORs of subclinical CVD with and without microalbuminuria [38]. In their analysis of type 2 diabetic patients, microalbuminuria was significantly associated with $\mathrm{ABI}$, after adjustment for other covariates (OR 6.05; 95\% CI 1.6122.76). In another study with 290 type 2 diabetic patients, Tseng et al. [39] found that patients with PAD had a significantly higher $\ln (\mathrm{ACR})$ value than those without $\mathrm{PAD}$, and $\ln (\mathrm{ACR})$ was correlated with $\mathrm{ABI}(\mathrm{r}=-0.198$; $\mathrm{p}<$ 0.01). After adjustment for other CV risk factors, albuminuria was significantly associated with PAD (OR 2.54; 95\% CI 1.05-6.17 for normoalbuminuria vs. microalbuminuria and OR 5.86; 95\% CI 1.76-19.52 for normoalbuminuria vs. macroalbuminuria).

In our study, albuminuria was associated with PAD, but not carotid plaque or CCA-IMT. We presently have no explanation for this difference between the three vessel structure markers. A possible explanation is that although they are similar, they are not identical factors [40]. Peripheral artery plaques are relatively more stable and less likely to rupture [40] and PAD may have a microvascular component [41] and shared pathogenesis with retinopathy [42].

One possible explanation for the association between atherosclerosis and albuminuria is that vascular endothelial damage can cause atherosclerosis and albuminuria [43]. Endothelial dysfunction changes the endothelial properties and has structural and functional effects on the target vessel, including changes in hemostasis and fibrinolysis, vasomotor activity, permeability to macromolecules, leukocyte adhesion, and vascular smooth muscle cell proliferation [44]. Thus, endothelial dysfunction plays a key role in the initiation and progression of atherosclerosis [44]. Additionally, endothelial dysfunction affecting the glomerular basement membrane could modify glomerular barrier permeability, leading to albumin excretion into the urine [45].
Lipid profiles may also affect the association between IMT, carotid plaque, and albuminuria. Glomerular cells possess LDL receptors, and mesangial and glomerular epithelial cells can internalize atherogenic lipoproteins via receptor-mediated and non-receptor-mediated mechanisms [46]. The infiltration of atherogenic lipoproteins into the glomerular endothelium and mesangial cells can trigger a series of events, such as adhesion molecule expression, monocyte chemoattractant production, and the release of reactive oxygen species, which lead to glomerular injury [47]. Thus, we suggest that lipid profiles, such as LDL cholesterol, might confound the association between atherosclerosis and albuminuria. In the present study, examination of the crude OR and sex-age-adjusted OR showed that albuminuria was marginally associated with carotid plaque; however, this association disappeared when additionally adjusted for other covariates. When we assessed the effects of other covariates, respectively, LDL cholesterol exerted a major influence on this disassociation.

This study possesses several limitations. First, we did not observe the incidence of CVD and PAD events, but, instead, attempted to predict the incidence of CVD and PAD, based on such indicators as IMT, carotid plaque, baPWV, and ABI, known surrogates for atherosclerosis and PAD. Second, we used a single morning spot urine sample to assess microalbuminuria, instead of timed urine collections, which would have been preferable. However, early morning urine provides a good estimate of 24-hour urinary albumin excretion rates, and a urinary ACR $>3.0 \mathrm{mg} / \mathrm{mmol}$ is associated with an albumin excretion rate $>30 \mathrm{mg} / \mathrm{min}$, with high sensitivity and specificity [48]. Despite its limitations, this study is valuable in that we evaluated the association between various subclinical atherosclerosis phenotypes, such as CCAIMT, carotid plaque, ABI, and baPWV, in a single population.

In conclusion, albuminuria was significantly associated with baPWV and PAD, but not carotid plaque or CCA-IMT in Korean type 2 diabetic patients. Additional prospective studies are required to further evaluate the mechanisms underlying these associations, and lack of associations, in Korean patients with type 2 diabetes. 


\section{References}

>1 Wild S, Roglic G, Green A, Sicree R, King H: Global prevalence of diabetes - estimates for the year 2000 and projections for 2030. Diabetes Care 2004;27:1047-1053.

$\checkmark 2$ Pan WH, Cedres LB, Liu K, Dyer A, Schoenberger JA, Shekelle RB, et al: Relationship of clinical diabetes and asymptomatic hyperglycemia to risk of coronary heart disease mortality in men and women. Am J Epidemiol 1986;123:504-516.

-3 Stamler J, Vaccaro O, Neaton JD, Wentworth D: Diabetes, other risk factors, and 12-year cardiovascular mortality for men screened in the Multiple Risk Factor Intervention Trial. Diabetes Care 1993;16:434-444.

4 Gerstein HC, Mann JFE, Yi Q, Zinman B, Dinneen SF, Hoogwerf B, et al: Albuminuria and risk of cardiovascular events, death, and heart failure in diabetic and nondiabetic individuals. JAMA 2001;286:421-426.

5 Wachtell K, Ibsen H, Olsen MH, BorchJohnsen K, Lindholm LH, Mogensen CE, et al: Albuminuria and cardiovascular risk in hypertensive patients with left ventricular hypertrophy: the LIFE study. Ann Intern Med 2003;139:901-906.

6 Dinneen SF, Gerstein HC: The association of microalbuminuria and mortality in non-insulin-dependent diabetes mellitus: a systematic overview of the literature. Arch Intern Med 1997;157:1413-1418.

7 Mathiesen ER, Ronn B, Storm B, Foght H, Deckert T: The natural course of microalbuminuria in insulin-dependent diabetes: a 10year prospective study. Diabet Med 1995;12: 482-487.

$>8$ Touboul PJ, Elbaz A, Koller C, Lucas C, Adrai V, Chedru F, et al: Common carotid artery intima-media thickness and brain infarction. GENIC case-control study. Circulation 2000;102:313-318.

-9 O'Leary DH, Polak JF, Kronmal RA, Manolio TA, Burke GL, Wolfson SK J: Carotid artery intima and media thickness as a risk factor for myocardial infarction and stroke in older adults. N Engl J Med 1999;340:14-22.

>10 Grobbee DE, Bots ML: Carotid intima-media thickness as an indicator of generalized atherosclerosis. J Intern Med 1994;236:567573.

-11 Androulakis AE, Andrikopoulos GK, Richter DJ, Tentolouris CA, Avgeropoulou CC, Adamopoulos DA, et al: The role of carotid atherosclerosis in the distinction between ischemic and non-ischemic cardiomyopathy. Eur Heart J 2000;21:919-926.

12 Golledge J, Greenhalgh RM, Davies AH: The symptomatic carotid plaque. Stroke 2000;31: 774-781.

13 Resnick HE, Lindsay RS, McDermott MM, Devereux RB, Jones KL, Fabsitz RR, et al: Relationship of high and low ankle brachial index to all-cause and cardiovascular disease mortality: the Strong Heart Study. Circulation 2004; 109:733-739.
14 Blacher J, Asmar R, Djane S, London GM, Safar ME: Aortic pulse wave velocity as a marker of cardiovascular risk in hypertensive patients. Hypertension 1999;33:11111117.

15 Yokoyama H, Hirasawa K, Aoki T, Ishiyama M, Koyama K: Brachial-ankle pulse wave velocity measured automatically by oscillometric method is elevated in diabetic patients with incipient nephropathy. Diabet Med 2003;20:942-945.

16 Tkac I, Molcanyiova A: Fibrinogen and albuminuria are related to the presence and severity of peripheral arterial disease in women with type 2 diabetes mellitus. Angiology 1997;48:715-719.

17 Stefan A, Fagerberg B: Microalbuminuria and intima-media thickness of the carotid artery in clinically healthy men. Atherosclerosis 2002;164:161-166.

18 Kramer H, Jacobs DR Jr, Bild D, Post W, Saad MF, Detrano R, et al: Urine albumin excretion and subclinical cardiovascular disease. Hypertension 2005;46:38-43.

19 Friedewald WT, Levy RI, Fredrickson DS: Estimation of the concentration of low-density lipoprotein cholesterol in plasma, without use of the preparative ultracentrifuge. Clin Chem 1972;18:499-502.

20 Lustgarten JA, Wink RE: Simple rapid kinetic method for serum creatinine measurement. Clin Chem 1972;18:1419.

21 Levey AS, Bosch JP, Lewis JB, Greene T, Rogers N, Roth D, et al: A more accurate method to estimate glomerular filtration rate from serum creatinine: a new prediction equation. Ann Intern Med 1999;130:461-470.

22 Ishizakaa Y, Ishizaka N, Tani M, Toda A, Toda EI, Koike K, et al: Relationship between albuminuria, low eGFR, and carotid atherosclerosis in Japanese women. Kidney Blood Pressure Res 2008;31:164-170.

23 Freedman BI, Langefeld CD, Lohman KK, Bowden DW, Carr JJ, Rich SS, et al: Relationship between albuminuria and cardiovascular disease in type 2 diabetes. J Am Soc Nephrol 2005;16:2156-2161.

-24 Mathiesen ER, Rønn B, Storm B, Foght H, Deckert T: The natural course of microalbuminuria in insulin-dependent diabetes: a 10 year prospective study. Diabet Med 1995; 12 : 482-487.

25 Deckert T, Feldt-Rasmussen B, Borch-Johnsen K, Jensen T, Kofoed-Enevoldsen A: Albuminuria reflects widespread vascular damage. The Steno hypothesis. Diabetologia 1989;32:219-226.

26 Finn AV, Kolodgie FD, Virmani R: Correlation between carotid intimal/medial thickness and atherosclerosis. A point of view from pathology. Arterioscler Thromb Vasc Biol 2010;30:177-181.
27 Megnien JL, Simon A, Gariepy J, Denarie N, Cocaul M, Linhart A, et al: Preclinical changes of extracoronary arterial structures as indicators of coronary atherosclerosis in men. J Hypertens 1998;16:157-163.

28 Ishimura E, Taniwaki H, Tsuchida T, Obatake N, Emoto M, Shoji T, et al: Urinary albumin excretion associated with arterial wall stiffness rather than thickness in type 2 diabetic patients. J Nephrol 2007;20:204211.

29 Safar ME, London GM: Structure fTCCoA, function: therapeutic studies and arterial stiffness in hypertension: recommendations of the European Society of Hypertension. J Hypertens 2000;18:1527-1535.

30 Laurent S, Boutouyrie P, Asmar R, Gautier I, Laloux B, Guize L, et al: Aortic stiffness is an independent predictor of all-cause and cardiovascular mortality in hypertensive patients. Hypertension 2001;37:1236-1241.

31 Munakata M, Ito N, Nunokawa T, Yoshinaga K: Utility of automated brachial ankle pulse wave velocity measurements in hypertensive patients. Am J Hypertens 2003;16: 653-657.

32 Sugawara J, Hayashi K, Yokoi T, Cortez-Cooper MY, DeVan AE, Anton MA, et al: Brachial-ankle pulse wave velocity: an index of central arterial stiffness? J Hum Hypertens 2005;19:401-406

33 Munakata M, Nunokawa T, Tayama J, et al: Brachial-ankle pulse wave velocity as a novel measure of arterial stiffness: present evidence. Curr Hypertens Rev 2005;1:223234.

-34 Dart AM, Kingwell BA: Pulse pressure - a review of mechanisms and clinical relevance. J Am Coll Cardiol 2001;37:975-984.

$>35$ O’Rourke MF, Safar ME: Relationship between aortic stiffening and microvascular disease in brain and kidney: cause and logic of therapy. Hypertension 2005;46:200-204.

-36 Knight EL, Kramer HM, Curhan GC: Highnormal blood pressure and microalbuminuria. Am J Kidney Dis 2003;41:588-595.

- 37 Kohara K, Tabara Y, Tachibana R, Nakura J, Miki T: Microalbuminuria and arterial stiffness in a general population: the Shimanami Health Promoting Program (J-SHIPP) Study. Hypertens Res 2004;27:471-477.

$>38$ Jie JC, Joshua IB, Do P, Teri AM, Bruce MP, Lewis K, et al: The association of microalbuminuria with clinical cardiovascular disease and subclinical atherosclerosis in the elderly: the Cardiovascular Health Study. Atherosclerosis 2006;187:372-377.

$>39$ Tseng CH, Chong CK, Tseng CP, Tai TY: The association between urinary albumin excretion and ankle-brachial index in elderly Taiwanese patients with type 2 diabetes mellitus. Age Ageing 2008;37:77-82. 
-40 Forrest KY, Becker DJ, Kuller LH, Wolfson SK, Orchard TJ: Are predictors of coronary heart disease and lower-extremity arterial disease in type 1 diabetes the same? A prospective study. Atherosclerosis 2000;148: 159-169.

-41 Blumenthal HT, Berns AW, Goldenberg S, Lowenstein PW: Etiologic considerations in peripheral vascular diseases of the lower extremity with special reference to diabetes mellitus. Circulation 1966;33:98-106.

-42 Adler AI, Stevens RJ, Neil A, Stratton IM, Boulton AJM, Holman RR: UKPDS 59: Hyperglycemia and other potentially modifiable risk factors for peripheral vascular disease in type 2 diabetes. Diabetes Care 2002; 25:894-899.
43 Stehouwer CDA: Endothelial dysfunction in diabetic nephropathy: state of the art and potential significance for non-diabetic renal disease. Nephrol Dial Transplant 2004;19: 778-781.

44 Stehouwer CDA, Smulders YM: Microalbuminuria and risk for cardiovascular disease: analysis of potential mechanisms. J Am Soc Nephrol 2006;17:2106-2111.

45 Deen WM: What determines glomerular capillary permeability? J Clin Investig 2004; 114:1412-1414.
46 Samuelsson O, Mulec H, Knight-Gibson C, Attman P, Kron B, Larrson R, et al: Lipoprotein abnormalities are associated with increased rate of progression of human chronic renal insufficiency. Nephrol Dial Transplant 1997;12:1908-1915.

47 Kamanna VS, Roh DD, Kirschenbaum MA: Hyperlipidemia and kidney disease: concepts derived from histopathology and cell biology of the glomerulus. Histol Histopathol 1998;13:169-179.

-48 Hutchison A, O’Reilly D, MacCuish A: Albumin excretion rate, albumin concentration, and albumin/creatinine ratio compared for screening diabetics for slight albuminuria. Clin Chem 1988;34:2019-2021. 